\title{
An Exploration of Smartphone Based Mobile Money Applications in Pakistan
}

\author{
Samia Ibtasam \\ University of Washington \\ Seattle, Washington, USA \\ samiai@cs.washington.edu \\ Jennifer Webster \\ University of Washington \\ Seattle, Washington, USA \\ jenniweb@cs.washington.edu
}

\author{
Hamid Mehmood \\ Information Technology University \\ Lahore, Pakistan \\ mscs15057@itu.edu.pk \\ Sarah Yu \\ University of Washington \\ Seattle, Washington, USA \\ sarahyu@cs.washington.edu
}

\author{
Lubna Razaq \\ Information Technology University \\ Lahore, Pakistan \\ lubna.razaq@itu.edu.pk \\ Richard Anderson \\ University of Washington \\ Seattle, Washington, USA \\ anderson@cs.washington.edu
}

\begin{abstract}
Worldwide, two billion people remain unbanked, the majority of whom reside in resource-constrained environments. While banks have limited reach due to high overhead costs of physical expansion, the global increase in mobile penetration has created opportunities to serve the unbanked using mobile-based Digital Financial Services (DFS). However, access to mobile applications alone is insufficient to ensure their trial, adoption, or continued usage. In this paper, we report a three phase learnability evaluation $(\mathrm{N}=118)$ of smartphonebased mobile wallet applications conducted in Pakistan. We discuss ways in which previous exposure or domain knowledge improve learnability, and we recommend that metrics for learnability should include effectiveness and help sought, independent of usability. We also identify DFS adoption opportunities such as user readiness, interface improvements, and women's independence. All of these opportunities stem from awareness and understanding of relevance, which in our case occurred as a consequence of exposure to the application under evaluation.
\end{abstract}

\section{CCS CONCEPTS}

- Human-centered computing $\rightarrow$ User studies; Usability testing;

\section{KEYWORDS}

ICTD; Mobile Money; Digital Financial Services; Mobile Wallet; Financial Inclusion;

\section{ACM Reference format:}

Samia Ibtasam, Hamid Mehmood, Lubna Razaq, Jennifer Webster, Sarah Yu, and Richard Anderson. 2017. An Exploration of Smartphone Based Mobile Money Applications in Pakistan. In Proceedings of ICTD, Lahore, Pakistan, November 16-19, 2017 (ACM ICTD '17), 11 pages.

https://doi.org/10.1145/3136560.3136571

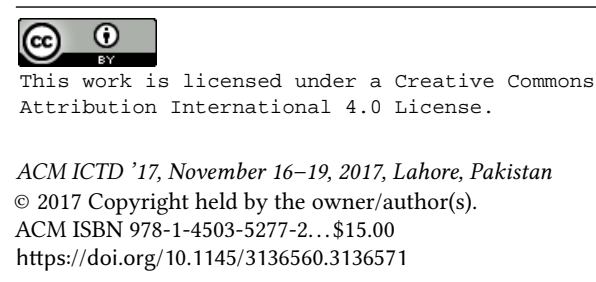

\section{INTRODUCTION}

Reliable access to financial services has been linked to overcoming poverty, narrowing income inequalities, and enabling economic growth [10]. Basic financial services include sending money, making or receiving payments, and saving or borrowing money. Provision of these services requires a customer to have an account at a formal financial institution.

The close link between financial well-being and access to the full suite of financial services is why financial inclusion, or the expansion of the number of individuals included in the financial system, has become a priority in reducing poverty and income gaps within and between countries. Today, approximately $38 \%$ of adults worldwide remain unbanked, meaning they do not have an account at a financial institution [3]. Typically, banks operate through physical branches and require a significant investment of capital for infrastructure and operations. Such investment means that low-income segments of the populace are not served due to their low profit margins.

Mobile money (or branchless banking), which provides financial services using the widespread cellular and distribution network of mobile operators, has been pursued as a means to expand financial inclusion. While the services associated with a financial account are rendered over a mobile device connected to their mobile wallet account, customers deposit and withdraw cash from these accounts through retailers or mobile money agents. In this paper, we focus on smartphone-based mobile money applications as a means of increasing financial inclusion. In particular, we explore the learnability of smartphone-based mobile money applications and their adoption by people in urban, semi-urban and rural areas of Pakistan while paying particular attention to rural users across multiple iterations.

After initial access, the ability to understand and learn applications plays an important role in the adoption and continued use of mobile money services as well as in enabling customers to fully utilize the numerous services within mobile wallets. There are two types of learnability: initial and extended. Initial learnability is defined as the user's ability to understand how to use a service or system right away. The other, extended learning, refers to a longer term learning of a service or system over time [13]. In this study, we concentrate on initial learnability because the trial, adoption, and uptake of mobile money applications is dependent on initial learnability. 
To understand the initial learnability of smartphone-based mobile money applications, we conducted a three-phase evaluation with participants from both urban and rural, semi to low-literate and illiterate populations in the province of Punjab, Pakistan. Since initial learnability is based on first time interaction, we selected participants who previously had not used any smartphone-based mobile money applications. To ensure initial learnability, we included each participant in only one phase of the study. All of the participants selected for these studies were first-time users of the smartphone applications being tested, in order to isolate learnability as initial learnability.

We ask the following questions:

- How learnable are the current mobile money applications available in Pakistan for users with low literacy?

- Would low-literate users prefer mobile wallets over conventional payment methods?

- What additional factors will enable self-learning and adoption of these services by users?

- How can we differentiate the evaluation of learnability with usability?

To answer these questions, we administered a three-phase, taskbased evaluation of one real and two prototype applications. We conducted guided field interviews with a total of 118 participants. First, we investigated a smartphone-based mobile wallet, EasyPaisa, offered by Telenor, a telecommunication provider in Pakistan. During the first phase, we observed difficulties faced by low-literate participants in using this application. In the second phase, we shifted our investigation from the preexisting mobile wallet to an application prototype based on a UI toolkit specifically design for low-literate users in Pakistan. We used two versions of this application; one was a protoype based on the default toolkit and the second had the added feature of side-by-side audio help. We discovered that audio was particularly helpful for low-literate populations. Therefore in the third phase, we tested a modified version of the prototype with audio help.

We found that side-by-side audio increases low-literate users' ability to learn the system and complete tasks. However, we identified other factors, besides usability and the application's interface, that affected users' comprehension and possible adoption of the system. These factors were predominantly related to awareness and understanding of the domain of digital payments. We observed an increase in participants' understanding of the benefits of mobile money applications. We noted several concerns regarding women's use of technology such as limited ownership and access to mobile phones. We also learned from our interviews with female participants that mobile money applications may be an opportunity for increased independence.

\section{RELATED WORK}

Earlier studies have addressed improving the usability of mobile interfaces for low-literate users. However, none have focused on improving the learnability of systems and the resulting uptake of services from the perspective of $\mathrm{HCI}$ in the context of resource constrained regions.

Usability and Learnability. Usability is defined as the extent to which a product can be used by its users to attain specified goals with effectiveness, efficiency, and satisfaction in a specified context of use [23]. Learnability is considered an important component of usability. However, there is no uniform definition of learnability in the HCI literature [13]. For example, in some studies learnability is defined as the ease with which a user can learn and start using a system [22] [13] whereas, Vatrapu et al. [28] refers to learnability as the evaluation of a particular system in terms of the learning process of the users of that system. Dix et al. [11] define learnability in terms of the ease of a system for users to accomplish basic tasks when using the system for the first time.

Alhough there is debate over the boundaries between learnability and usability, learnability is often considered an attribute of usability [15]. Nielson [21] refered to learnability as a fundamental attribute of usability because the first experience that users might have with a product is that of learning to use it. In our work, we define learnability as the ease with which users are able to learn to use the system, without requiring any external guidance or support, and achieve the task they wish to perform.

Learnability is foundational to sustained use because users who are unable to learn how to use an application within the first few minutes will switch to another application [13]. With this assumption, we argue that difficulties in a mobile money application's learnability may result in the user defaulting to existing modes of payment or cash payments because of their ease of use or familiarity.

Interface design plays an important role in the overall learnability of a system. Difficulties in interacting with the interface, at any point, can decrease overall learnability. Leung et al. [17] studied learnability of mobile applications for older adults and suggested three approaches for improving device learnability: improve graphical icons, create multi-layered interfaces, and augment the mobile interface.

Smartphone Interfaces for Mobile Money. In 2009, Medhi et al. [19] studied mobile money user interfaces in resource-constrained economies. The study focused on feature phones given the low penetration of mobile phones, especially smartphones. They recommended text-free designs over text-based designs and help on every screen to enable illiterate and novice users to navigate the system without external assistance. They argued that mobile ownership is associated with wealthier households, a fact that has changed since 2009. In Pakistan alone, the cellular teledensity has increased from $58.2 \%$ in 2009 to $70.8 \%$ in 2017 [24] . Smartphones are expected to grow to $50 \%$ of the subscriber base by 2020 compared to $16.6 \%$ in 2015 [26]. The services being analyzed by Medhi were text-based UI over USSD and SIM toolkit applications. Today, smartphone applications have become one of the mainstream media to provide services; all mobile money providers in Pakistan have applications.

Organizations such as the Consultative Group to Assist the Poor (CGAP) have looked into the design of smartphone interfaces for mobile money applications [6]. They argue that smartphones are likely to become the main force of expanding the mobile money user base and that smartphone interfaces have an important impact on the uptake, adoption, and retention of mobile money applications.

Many have researched speech, audio, video, and graphical guidance for low-literate users in smartphone interfaces as well. Video Kheti [8] recommended using both audio and graphical interfaces. 
However, they also found that multi-modal interfaces do not help low-literate users overcome issues related to lack of education. Graphical symbols and photos are excellent for output and convey large amounts of non-linguistic information. However, they introduce the risk of ambiguity and confusion regarding the meaning of images. Mendoza et al. [20], in addition to discussing user frustrations and user error categories, argued that the causes of novice users' frustrations represent "barriers to entry" rather than fundamental problems with application usability.

\section{MOBILE PHONES AND FINANCIAL INCLUSION}

Given the global rise in mobile phone and internet availability, financial services provided through mobile phones offer a promising solution for financial inclusion for those who have not had the opportunity to access branch banking networks. Efforts are ongoing to leverage mobile phone penetration to expand financial services as a means to decrease the unbanked population.

We focus on mobile applications and their learnability not only for the growing ubiquity of mobile phone and internet access, but also for their specific benefits to financial inclusion. Mobile phones reduce the geographical barrier of having to travel to bank locations, often located in only the most populous areas. Transacting and keeping money in these applications introduces a different level of privacy and security in contrast to traditional methods of physical cash storage or money transfer (i.e., money bus or van services). Using mobile money applications reduces the cost of money transactions, and the service is relatively inexpensive because it utilizes existing ownership of phones. With reduced cost and using existing infrastructure, the goals of financial inclusion are more readily obtained.

We also focus on smartphones, rather than all mobile phones because of growing smartphone penetration rates and the proliferation of $3 \mathrm{G}$ and $4 \mathrm{G}$ networks in Pakistan and elsewhere in the world. While mobile phones, in general, are a strong medium for financial services, smartphones offer the possibility of more capabilities and computational power.

Digital Financial Services (DFS) are generally defined as financial services delivered through digital channels that reduce the limitations posed by high costs and physical networks [18]. Some popular forms of DFS include Internet Banking, Mobile Wallets and Over-The-Counter (OTC) transactions. While there are many forms and nuances, especially depending on the specific geographical and cultural context, for our purposes we will define and differentiate between Mobile Wallets and OTC transactions. Mobile Wallets are basic financial accounts, often but not always, housed by banks or telecommunications operators that are accessed and maintained by an individual through their mobile device. This is in contrast to OTC transactions, which process a transfer through an in-person visit to an agent's shop. On the receiving end, the recipient takes a code and identification document to withdraw that money from another agent's shop [7].

OTC transactions have become a popular way of sending money through local shops in Pakistan. Customers often prefer the human to human interaction rather than using their phones as you would with a mobile wallet. However, OTC transactions do not translate to financial inclusion because they do not require the customer to have an account at a financial institution through which a customer can be provided services of payments, savings, lending, and insurance. Opening an account is the first step in creating a financial history for the customer, which can then be used to borrow money. Hence, for all practical purposes, shifting customers from using OTC transactions to opening and utilizing mobile wallet accounts is essential for financial inclusion as well as the associated economic uplift.

We chose to focus our work on Pakistan and the mobile money applications available there because Pakistan already has a wellestablished technological infrastructure including a smartphone based app ecology. However, although Pakistan is the sixth most populous country in the world with an estimated population of 186 million, there has been a low uptake of mobile money applications [26]. Likewise, Pakistan presents an interesting case study in that certain barriers to mobile money uptake are present even with the robust technological infrastructure. These barriers can include a number of variables such as income, literacy, gender, trust, and isolation. Determining how these variables affect mobile money application uptake, usability, and learnability, the results could potentially be generalizable to other regions outside of Pakistan.

An overview of the prerequisites for providing Digital Financial Services (DFS) in Pakistan reveals that it has all of the factors needed for their successful introduction. After the advent of $3 \mathrm{G}$ and $4 \mathrm{G}$ services in 2013, Pakistan's smartphone and mobile Internet penetration rates have been steadily increasing and it is estimated that $16.6 \%$ of mobile subscribers in Pakistan own a smartphone [26].

Pakistan has a strong national identification system covering $90 \%$ of Pakistan's population. It is backed by a robust biometric database and formalized through government issued identification cards known as Computerized National ID Card (CNIC) [26]. The presence of a strong central identification system facilitates fulfillment of the first step toward customer onboarding (i.e., Know Your Customer (KYC) requirements imposed by financial services regulations). The State Bank of Pakistan has introduced many mobile money friendly policies such as [5] the facilitation of lower KYC requirement accounts, also known as Level-Zero accounts [4], in the Branchless Banking regulation for mobile money. Opening a LevelZero mobile money account requires entering a customer's name and CNIC number that is verified against the National Database and Registration Authority (NADRA) database [27].

\section{FIELD WORK}

Our work consists of three iterations (described as Phases One, Two, and Three). Phase One examined the EasyPaisa mobile wallet offered by Telenor $(\mathrm{N}=30)$. Based on the findings from Phase One we tested a non-functional prototype of a mobile wallet that was developed using the wireframes of Karandaaz ( $\mathrm{N}=48$ ). After identifying issues from Phase Two, we made additional adjustments for Phase Three $(\mathrm{N}=40)$.

For our field research we partnered with Akhuwat, the largest non-profit interest-free micro finance institution in Pakistan. As of March 2017, Akhuwat had an outstanding loan portfolio of PKR 10 billion (USD 95 million) in outstanding loans. Akhuwat was 
established in 2001 and has an existing network of 613 branches across 200+ cities in Pakistan [2].

Akhuwat focuses on providing services for low-income individuals who might not be catered to by interest-based micro finance banks, which was the same criteria we used for determining potential participants. Our partnership with Akhuwat facilitated identifying participants since they had already established relationships at our research sites.

The participants in all three phases of the study had diverse occupations including local shop keepers, factory workers, daily wage laborers, security guards, vegetable vendors, small farmers, rickshaw and van drivers, small home-based business owners, university students, and housewives. table 1 shows participant details from all three phases. Participants were divided into four categories based on their literacy level: $\mathrm{I}=$ illiterate, $\mathrm{P}=$ Primary (1-5 standards), $\mathrm{M}=$ Middle to Matriculation (8-10 standards), $\mathrm{G}=$ Intermediate or Graduation ( $>12$ standards) and age group: $\mathrm{A}=(<25$ years $), \mathrm{B}=(<$ 35 years), $\mathrm{C}=(<45$ years $), \mathrm{D}=(<55$ years $)$.

Each session took an average of 40-45 minutes per participant. The studies were conducted in local languages (Urdu and Punjabi) depending on the preference of the respondents. The interviews were audio recorded with the informed consent of the participants. Interviews were conducted based upon the comfort and availability of the participants. Some were interviewed in their homes, some in a small local tea stall, and the majority of the interviews in Phases Two and Three were conducted in branch offices of our field partner, Akhuwat.

The three iterative studies described in this paper comprised of the following three steps.

Step 1: Pre-task Interview. The Pre-task interview consisted of questions about basic demographics, employment, network selection, phone ownership and usage level, familiarity with smartphones, existing practices and sources of saving, sending and receiving money, ownership and use of bank accounts, previous banking experience, interaction with Automated Teller Machines (ATMs), knowledge or awareness about OTC transactions, mobile and internet banking, and mobile wallets.

Step 2: Task Based Learnability Evaluation. To evaluate the learnability of the mobile wallet application, we selected a set of tasks for the participants to perform. Despite all participants being first-time users of the respective mobile money application, we did not provide any demos, trial runs, or tutorial videos of the task. This was to isolate the effects of initial learnability. Each participant was given a verbal introduction to the application and the tasks they had to perform (five tasks for Phase One, one task for Phase Two, and one for Phase Three). We evaluated learnability in terms of effectiveness (i.e., were participants able to do what they intended to do on their own?), help-seeking (i.e., how much external help or guidance was sought or required by the participants), and proficiency (i.e., were participants able to complete the tasks?). All of the inputs such as phone number, CNIC (Computerized National ID Card) number, and PIN codes required to perform the task were provided to the participants on a piece of paper. This was done to ensure that they did not enter any real personal information [16] and also to avoid any hesitation caused by requiring such data. Participants were provided a smartphone with the application installed on it to complete the given tasks. The devices used in the study were Android devices with 6-inch screens that ran Android version 6.0.

Step 3: Post-task Feedback. After the participants had performed the tasks and interacted with the mobile money applications, we sought feedback from the participants about the need for help within the application, problems they faced while using the applications, and their recommendations for improvements. Participants were also questioned about the need for training to use the application and their preferred ways of learning.

Learnability Evaluation Methodology: Coaching Methods \& Question Asking. Learning and understanding is significantly affected by introduction and guidance provided during task-based exercises. Because of this, we prepared and provided consistent answers for guidance to all participants. We considered question-suggestion and question-asking [13] coaching methods. Question-asking requires answering the users based upon user queries, whereas in question-suggestion, the coach asks questions based on the user's reactions and time elapsed (e.g. 'What are you trying to do here?').

We decided to go with question-asking to ensure uniformity of coaching to all users, irrespective of the coach's understanding of the user's learning. We had a coach and an observer for each interview. The coach was supposed to help with answering the questions and providing guidance while the observer noticed and recorded participants physical and verbal reactions.

\section{PHASE ONE - AN EXPLORATION OF TELENOR'S MOBILE WALLET}

We began working with a preexisting mobile wallet application from Telenor, one of five major telecommunications service providers in Pakistan. In 2016, Telenor's wallet, EasyPaisa, had the highest market share. The benefit to using a preexisting wallet was that we could easily select which tasks we wanted to test and we did not have to modify or build the application. However, EasyPaisa is an English-language only application and is not available in the local language, Urdu.

A total of 30 interviews were conducted in two locations over two and three days respectively. Ten interviews were conducted in Lahore, the second largest city of Pakistan, and the other 20 were conducted in Kamalia, a rural area 250 kilometers from Lahore.

\subsection{Task Description and Allocation}

The EasyPaisa mobile wallet offers over 15 major tasks. We selected a total of five tasks based upon their estimated frequency of need (like sending money) or the importance of the task to the overall operation of the mobile money application (e.g., changing the pin). The five tasks were:

- Change PIN - required navigating to the 'My Account' option, selecting 'Manage PIN', selecting 'Change PIN', entering existing PIN, and then entering new PIN twice to change the PIN

- Send Money to an EasyPaisa Account - required selecting the Ḿney Transfer' option. Then, on the transfer money screen, as shown in Figure 1 (left), you could select from methods of transferring money like from bank account, Easy Paisa, or 


\begin{tabular}{|l|l|l|l|l|l|l|}
\hline \multirow{2}{*}{ Gender } & \multicolumn{2}{c|}{$\begin{array}{c}\text { Phase 1: } \\
\text { Telenor App }\end{array}$} & \multicolumn{2}{c|}{$\begin{array}{c}\text { Phase 2: } \\
\text { Karandaaz App }\end{array}$} & \multicolumn{2}{c|}{$\begin{array}{c}\text { Phase 3: } \\
\text { Updated Karandaaz }\end{array}$} \\
\hline No.of Participants & Male & Female & Male & Female & Male & Female \\
\hline \multirow{5}{*}{ Age } & 20 & 10 & 32 & 16 & 29 & 11 \\
& A: 6 & A: 4 & A: 7 & A: 7 & A: 9 & A: 4 \\
& B: 9 & B: 3 & B: 12 & B: 5 & B: 10 & B: 6 \\
& C: 4 & C: 2 & C: 8 & C: 4 & C: 8 & C: 1 \\
& D: 1 & D: 1 & D: 5 & D: 0 & D: 2 & D: 0 \\
\hline \multirow{5}{*}{ Education } & I: 0 & I: 3 & I: 4 & I: 3 & I: 4 & I: 3 \\
& P: 4 & P: 1 & P: 4 & P: 0 & P: 5 & P: 3 \\
& M: 10 & M: 2 & M: 19 & M: 6 & M: 16 & M: 4 \\
& G: 6 & G: 4 & G: 5 & G: 7 & G: 4 & G: 1 \\
\hline \multirow{5}{*}{ Phone Ownership } & Feature: 9 & Feature: 4 & Feature: 19 & Feature: 7 & Feature: 17 & Feature: 8 \\
& Smart: 10 & Smart: 3 & Smart: 12 & Smart: 5 & Smart: 7 & Smart: 3 \\
& Both: 1 & Both: 0 & Both: 1 & Both: 3 & Both: 5 & Both: 0 \\
& None: 0 & None: 3 & None: 0 & None: 1 & None: 0 & None: 0 \\
\hline \multirow{2}{*}{ Locality } & Rural:14 & Rural: 6 & Rural: 20 & Rural: 3 & Rural: 25 & Rural: 6 \\
& Urban: 6 & Urban: 4 & Urban: 12 & Urban: 13 & Urban: 4 & Urban: 5 \\
\hline \multirow{2}{*}{ Previous OTC Usage } & Yes: 15 & Yes: 1 & Yes: 23 & Yes: 4 & Yes: 15 & Yes: 5 \\
& No: 5 & No: 9 & No: 9 & No: 12 & No: 14 & No: 6 \\
\hline \multirow{2}{*}{ Internet Access \& Usage } & Access: 16 & Access: 6 & Access: 26 & Access: 13 & Access: 19 & Access: 6 \\
& Usage: 12 & Usage: 4 & Usage: 15 & Usage: 15 & Usage: 15 & Usage: 4 \\
\hline \multirow{2}{*}{ Mobile Wallet Usage } & Yes: 2 & Yes: 0 & Yes: 2 & Yes: 0 & Yes: 7 & Yes: 0 \\
& No: 18 & No: 10 & No: 30 & No: 16 & No: 22 & No: 11 \\
\hline \multirow{2}{*}{ Bank Account Ownership } & Yes: 13 & Yes: 4 & Yes: 15 & Yes: 5 & Yes: 18 & Yes: 3 \\
& No: 7 & No: 6 & No: 17 & No: 9 & No: 11 & No: 8 \\
\hline
\end{tabular}

Table 1: User Profiles for All 3 Iterations

CNIC, then entering the receiver's phone number, and then entering the amount to be sent

- Send Money to Bank Account - required choosing the bank of the receiver from the list of banks, then entering account information, and the entering the amount to be sent.

- Sending Top Ups - required entering the amount to be sent, selecting the network operator of the recipient, and the the number to send the airtime to

- View Statements - required navigating to "My Account" and finding past transaction details

Participants were allowed to ask for help during the session, but only for Urdu translation of English words.

\subsection{Phase One Observations}

Evaluation of Telenor's mobile wallet revealed a close relationship between interface issues and learnability of the systems. Table 2 shows the overall task completion rate by participants. We found that higher literacy levels, which also correlated to higher previous smartphone ownership, were connected to higher completion rates by participants.

Participants reported issues with navigating the application's menus and sub-menus. This required applicants to explore before locating their desired option. For example, Figure 1 (right) illustrates one such interface. The red box at the bottom of the screen contained user options like 'My Account' and 'Store Locator'. Whereas, the yellow box in the center of the screen showed current features available to participants. There is also a hamburger icon in the top left corner, highlighted in the maroon box in Figure 1, which reveals yet another menu. The participants requested a simpler interface with less functionality on one page and no hidden menus.

In Telenor's mobile wallet, the participants had to enter all of the inputs for a particular task (like sending money) on a single screen as shown in Figure 1 (left). This increased the complexity and cognitive load for participants, and many became confused while entering multiple inputs on a single screen.

One participant described their experience as follows: "It was confusing due to the large number [of menus] and difficult due to the proximity of so many inputs on a single screen. Instead the interface should be like an ATM interface where the options are fixed and require much less input from the users and only one task on each screen to make it understandable."

In addition, the tab-based design made other tabs visible to the user at all times. Both of these features - multiple inputs on one screen and multiple menus - provide an overwhelming amount of information to the end-user and can impact the initial learnability by increasing the cognitive load on the user. The design community [6], recommends against both of these features.

Additional interface issues that potentially impacted usage by illiterate or low-literate users were the interface language and supplementing cues. Telenor's EasyPaisa mobile wallet was available only in English and contained limited graphical cues with with no 


\begin{tabular}{lllll}
\hline Task Name / Literacy & Illiterate & Primary and Under Primary & Middle to Intermediate & Graduation and Above \\
\hline Change PIN & $0 \%$ & $0 \%$ & $66 \%$ & $100 \%$ \\
Send Money to EasyPaisa Account & $0 \%$ & $40 \%$ & $100 \%$ & $100 \%$ \\
Send Money to Bank Account & $0 \%$ & $40 \%$ & $92 \%$ & $100 \%$ \\
Sending Topup & $0 \%$ & $40 \%$ & $100 \%$ & $100 \%$ \\
View Statements & $0 \%$ & $0 \%$ & $83 \%$ & $100 \%$ \\
\hline
\end{tabular}

Table 2: Literacy and Task Completion Rates for Phase 1 - Telenor Wallet

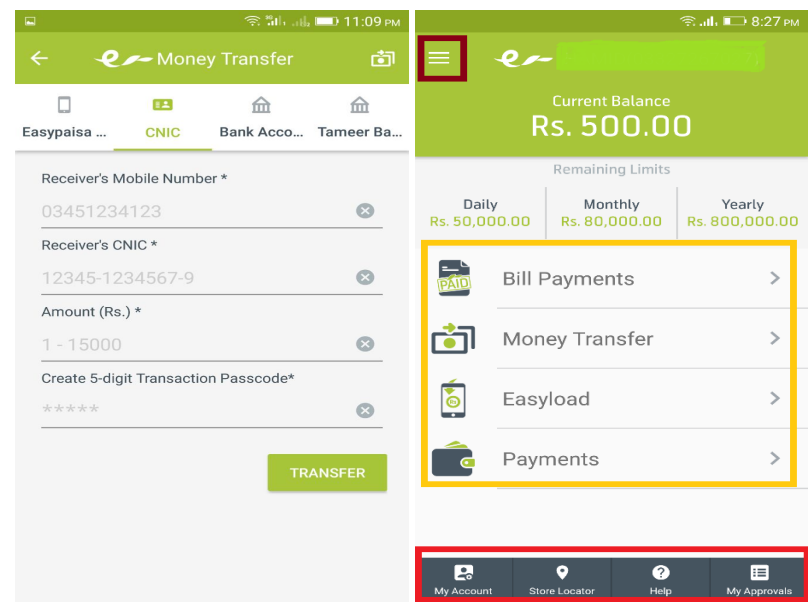

Figure 1: Screen showing Send Money screen with multiple sending options as tabs (send to bank account, EasyPaisa, CNIC) and multiple inputs within tabs (left), Screen with menu options, hamburger menu and lower menu options on one screen (right)

audio or video assistance. These factors made it extremely challenging for participants with limited or no English to interact with the application and complete tasks.

Because many participants struggled with the English only EasyPaisa application that lacked audio and visual assistance, we considered making changes to its UI to fix the issues and test again. However, we faced challenges in modifying the original application and editing its existing UI. Our solution for Phase Two was to work with a different application altogether.

\section{PHASE TWO - AN EXPLORATION OF THE KARANDAAZ UI AND OUR AUDIO HELP INTERVENTION}

At the end of the interview sessions for Phase One, we asked participants about what could increase learnability and what kind of help they would prefer to increase learnability. Most of the participants responded that they would prefer in-application help over individual help. For in-application help, the participants expressed that side-by-side audio within the applications would be an improvement. Thus, in Phase Two we decided to test these recommendations.

In July 2016, Karandaaz Pakistan, a not-for-profit development finance company, released a Design Toolkit [1] for smartphone mobile money applications. The design toolkit provided a user interface and user experience (UI/UX) prototype specifically focused on being inclusive of low-literate users. We decided to incorporate this UI because it already addressed many of the issues that we had discovered during Phase One. Unlike the applications produced by telecommunication companies, the Karandaaz Design Toolkit was an open source toolkit and easily accessible for use.

The Karandaaz prototype provides assistance primarily through text and graphics. It focuses on being accessible to low-literate users by providing an opening assistant, a built in on-boarding process, and consistent assistant help functions. The opening audio assistant is first introduced with the opening of the app and is available on every screen thereafter at the top right for general help. Each menu option is a graphical icon with text directly underneath it. The icon is intended to provide assisted recognition of the menu option. The starting menu offers four options from which the rest of the options can be navigated to through a combination of choices. However, the prototype is simply a collection of wireframes and is not in itself implemented or functional at the time of writing.

In this phase, we tested two applications, both of which we built using Karandaaz wireframes. One application had additional audio help intervention (hereafter differentiated as Karandaaz App and Audio App, with the Audio App containing the additional audio assistance). Unlike the EasyPaisa mobile wallet, which offered English language only, upon opening the Karandaaz App and the Audio App, participants were prompted to select from Urdu and English. The participants were randomly allocated either the Karandaaz App or the Audio App.

Phase Two was conducted over five days at five branch locations of Akhuwat and sometimes in the homes of participants. Three of the branch locations were in the peripheries of Lahore (peri-urban areas), whereas the remaining branches were in rural areas of Kasur.

Audio Help Intervention. For each Karandaaz implementation of graphical icons and an on-request assistant for contextual help, which could also provide speech upon clicking, we provided a per item speaker option for participants. If the images were ambiguous, participants could press the speaker button to hear audio help. In the Audio application, the built-in Karandaaz assistant was not introduced.

A speaker icon was provided against each interface item as shown in Figure 2 (left) and on tapping this speaker, an audio in Urdu language played explaining the functionality of the specific button. 


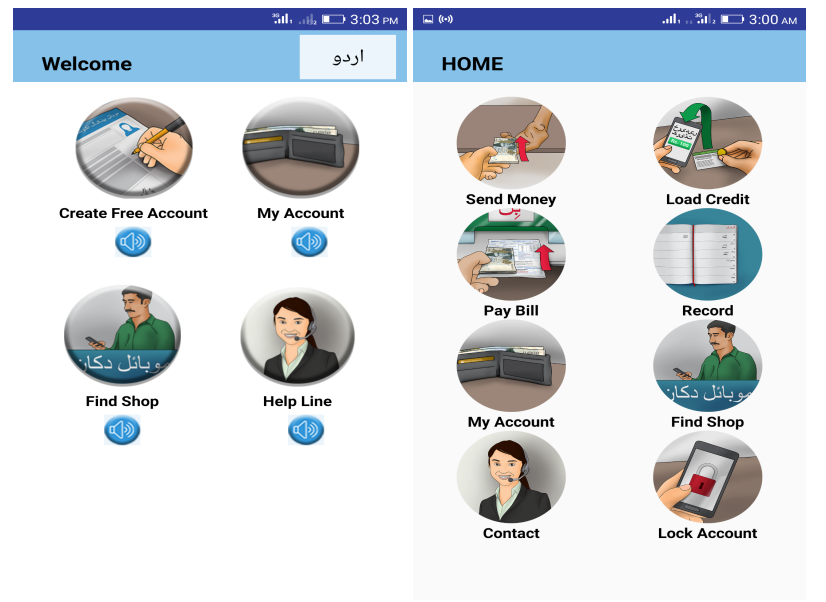

Figure 2: Speaker button against each option to provide audio help and option to select Urdu language (left), Home Screen with all options (right)

\subsection{Tasks Description and Allocation}

We implemented the login screens and the complete work flow for sending money to test learnability. The send money task had a total of 12 screens: three for login and nine for sending money. After logging in, the participants had to choose the 'Send Money' option from eight options within the Home screen (shown in Figure 2 right image). Upon selection and entering the sending money work flow, it was linear flow with a single task per screen. The participants could seek help by using the built-in Karandaaz assistant help in the Karandaaz Application or the audio help by using the audio buttons in the Audio Application (Figure 3 right). All of the other options such as paying bill, loading credit, etc. were available on the home screen but were not functional as shown in Figure 2 (right).

\subsection{Phase Two Observations}

Confusion between PIN code and Secret code. The Karadaaz UI Send Money step consisted of entering a PIN Code for authorization of the transaction and generating a Secret code that was to be shared with the recipient for cashing out the received amount at an agent location (the shopkeeper in the OTC model). This Secret code, along with other identification documents, had to be produced as an authentication token upon receipt. Despite having made previous OTC transactions, some of the respondents could not comprehend the term 'Secret code.' Upon probing, they mentioned that mobile money agents do not tell them about the secret codes nor do they ask them to generate any secret codes for recipients. The participants reported that the agents give them the "recipient's code" by writing it on a piece of paper and telling them that they should share it with the intended recipient.

Interface Issues. Although the Karandaaz UI catered to the interface issues faced during Phase One with Telenor wallet, we found some additional interface issues with the Karandaaz UI design.

The Karandaaz UI, as well as the walk through video of the toolkit [14], uses flat images as buttons. However, the participants considered those images only as explanatory pictures and not as buttons and thus avoided tapping them. This was primarily an issue on the Welcome Screen and the Home Screen Figure 2 (left,right), where the participants had to select from a number of options to go further.

Similarly, the Karandaaz UI did not use the native keyboards and instead designed an on-screen keyboard with ten digits and an $\mathrm{X}$ (for backspace) in the lower left corner of the keyboard Figure 3 (left). Participants, irrespective of their previous feature phone or smartphone usage and performance time, were unable to locate the $\mathrm{X}$ or identify it as a backspace option. This resulted in participants tapping the cancel button in the title bar or the back button at the bottom of the screen for even simple typing mistakes. Tapping the cancel button canceled the whole transaction and start all over again. Even tapping the back button would take them to the previous screen. This resulted in increased transaction time, multiplying the number of times a user would edit input text.

As mentioned in the Task Description Section, participants had to choose from multiple options (four on the Welcome screen and eight on Home screen). Even with the presence of graphics, the low-literate and illiterate participants were unable to identify which image corresponded to sending money. Participants became confused by Send Money, Pay Bill, and My Account, all of which contain some form of money or wallet on the icon.

The Karandaaz App had a help assistant function that can be invoked on screens by tapping on the title bar assistant icon. Before the tasks, participants were informed of the help assistant while being introduced to the tasks. However, none of the participants used the help assistant, despite the introduction. Some participants mentioned that they did not observe or notice it, while others said that they forgot about it. Improved discoverability of the help assistant will be necessary for utilization of the feature.

\section{PHASE THREE - AN EVALUATION OF THE MODIFIED APPLICATION}

Our Phase Two evaluation of two applications (the Karandaaz App and the Audio App) showed that urban and educated participants performed better while using the applications. In contrast, more learnability issues existed for the rural participants with comparatively lower education levels. Thus, during Phase Three, we focused our efforts on improving learnability for rural and low-literate populations. We used the same login and Send Money tasks for this phase. Phase Three was conducted over three days at Akhuwat branch locations in peri-urban areas of Lahore as well as rural areas of Kasur and Pattoki.

\subsection{Phase Three Application Modifications and Observations}

In Phase Three we made changes to the Audio App and evaluated participant use of the modified Audio App only. The changes were in the form of interface and workflow additions that enabled the participants to understand and learn the application. We modified the flat images to 3D icons to make them appear more like buttons [29]. The Figure 2 left image shows our 3D buttons in comparison to the Figure 2 right image showing the Karandaaz UI flat image buttons. However, participants in Phase Three still experienced difficulty in identifying the 3-D icons as buttons. 


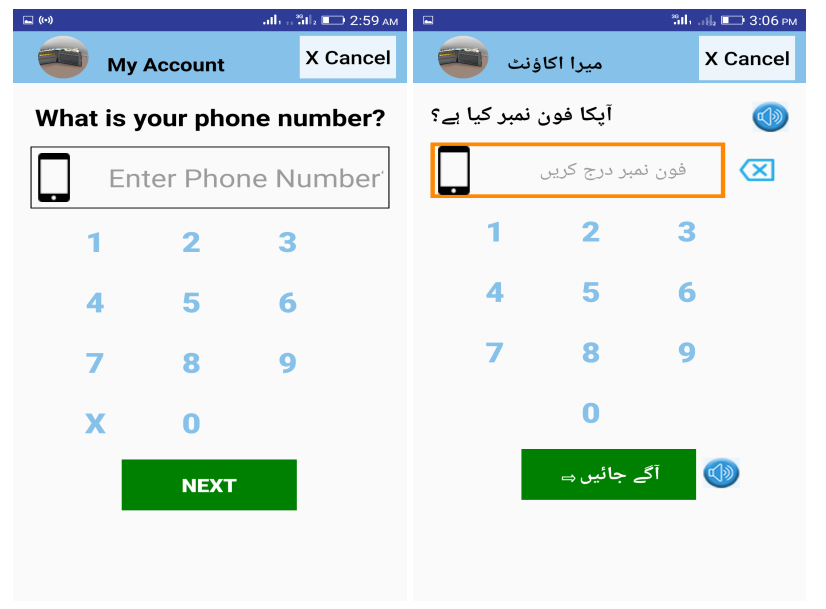

Figure 3: Backspace Button Phase 2 (left), Backspace location changed, Input Box highlighted while playing the relevant Audio, Speaker With each interface item (right)

To enable participants to better locate and identify the backspace button, we relocated the backspace button from the on-screen keyboard to the right side of the text box as shown in Figure 3. After making this change, all participants in Phase Three accurately located the backspace button (compare to 38\% from Phase Two). Consequently, we resolved the predominant error of tapping the cancel button and canceling the whole transaction simply for a typing mistake.

For illiterate participants, it was difficult to understand each screen upon screen load. Tapping speaker buttons enabled them to understand the features, but they also needed to understand the screen's function as a whole. Thus, to enable them to understand the required action for a certain screen, we introduced on-load audio prompts which explained the task to be performed on a given screen, such as entering phone number or entering PIN code, etc. One such prompt is "On this screen, enter the phone number of the person you wish to send money." Participants still had the option to use individual speaker buttons, but the on-load audio help quickly allowed participants to identify the task without the need for additional audio help from individual speaker buttons. This saved time for completing the tasks. The more literate participants also expressed that the on-load audio was beneficial.

Similarly, in the case of an incomplete or wrong input, an error message was displayed at the bottom of the screen. However, previously no audio output was available for the participants who could not read. Thus, we added error prompts to inform participants of the location and nature of error. For example "You entered incomplete phone number, Please enter the complete phone number consisting of 11 digits"

Some participants in Phase Two were unable to identify the input areas and were unable to relate the audio prompts to the appropriate input element. To overcome this issue, we highlighted the related input field while audio played. Highlighted (shown in Figure 3 (left)) input directed participants' attention to the relevant input areas and made identification easier.

Audio help embedded in various aspects of the application proved beneficial for illiterate and low-literate participants in completing the tasks, while simultaneously offered support for participants with weak eyesight. In addition to these, some of the literate participants also used the audio help. One literate participant mentioned that, "Written text might be misinterpreted but voice and audio explain it really well. Reading takes time and requires interpretation." It was also observed that participants were using audio help instead of icons to understand the functionality of buttons. Similarly, the addition of on-load audio prompts enabled users to identify the task within each screen, reducing both time to complete and error rates.

Out of the 40 participants in Phase Three, 18 people had trouble reading and referred to audio help. These 18 included seven respondents from the illiterate group, seven from the primary and under primary group, three from the middle group, and one from the matric group.

Coaching vs. Audio Help. During Phase Two, all participants completed the task, but the extent of help they received from the coach varied. Therefore, in Phase Three, we measured the extent to which participants asked the coach to help them understand what they needed to do. We measured this using a 4-point scale: no help, help in a few places, help in most places and help throughout the session. We found that on the whole, $50 \%$ of participants required no help during the session, $35 \%$ percent required help in a few places, $5 \%$ required help in most the places, and $10 \%$ required consistent help throughout the session. Most of the participants who did not ask for help from the coach were educated, so we further focused our analysis on illiterate and low-literate participants.

Table 3 shows that the majority of low-literate participants who were smartphone owners did not seek any help from the coach nor did they use audio help. It also shows that the majority of illiterate and low-literate participants referred to audio help to complete tasks and only asked the coach for help at a few places, regardless of their lack of exposure or ownership of smartphones. We can, therefore, conclude that smartphone ownership has a positive effect on the learnability of the application for low-literate users. We also conclude that the use of audio help makes it possible to complete the task with limited help for illiterate and low-literate users who have never owned or been exposed to smartphones.

\section{FINDINGS ACROSS ALL PHASES}

Along with the observations mentioned in each phase, we also observed the following commonalities across all phases.

Preferred Help for Learning. Out of the total 118 participants, $102(87 \%)$ mentioned the need for for some kind of help in the application. Out of 102 participants, 66 preferred audio help, 31 preferred video help, and the remaining five were in favor of help via call to a helpline. When asked about the preferred language for in-application help, 90 participants preferred Urdu, 7 preferred Punjabi, and 5 requested help in English. 85 participants preferred side-by-side assistance on each screen and 17 preferred assistance only on the start screen.

"Video is fast and I cannot comprehend and remember the entire video. I prefer side by side audio help." 


\begin{tabular}{lllll}
\hline Phone Ownership/Help Type(top) & No Help & Help at few places & Help at most of places & Consistent help \\
\hline Feature Phone and No Exposure to Smartphone & 2 & 9 & 0 & 3 \\
Feature Phone with Exposure to Smartphone & 1 & 1 & 1 & 1 \\
Smartphone owner & 4 & 1 & 0 & 0 \\
Referring to Audio help & 3 & 9 & 1 & 4 \\
Not referring to Audio help & 4 & 2 & 0 & 0
\end{tabular}

Table 3: Help requested from coach by low-literate participants - shown against previous feature or smart phone ownership and exposure (above) and shown against use of Audio help (below)

Using Time Taken as a Metric. Participants who used the audio help within the application were also the participants who successfully completed the tasks, irrespective of their educational level. However, all participants who used audio help took more time due to the added audio playing time. Average time taken by participants referring to audio help was 9.41 minutes, whereas the average time taken by participants not referring to audio help was 4.53 minutes. Thus, when discussing and calculating learnability, the time taken to complete tasks cannot be used as an effective metric.

Knowledge vs. Exposure to Mobile Money. The knowledge or understanding of mobile wallets is not synonymous with OTC transaction exposure. Out of 118 participants, 63 were using OTC transactions to send or receive money. Out of these 63, 11 participants were also using mobile wallets to send or receive money. We found that these participants were not familiar with the term mobile wallet. Only by probing through an explanation of the concept in the local language of the participant did we reveal that they were using mobile wallets. 31 of the participants did not know or understand the term mobile wallet. While three of these 31 had heard the word mobile wallet on a TV advertisement, they still did not understand what it was.

Previous exposure and experience of participants with OTC transactions or mobile wallets had an impact on the learnability of the application. In Phase Three, people who had sent money using OTC or a mobile wallet took 5 minutes 30 seconds on average to complete a transaction, while people who had never conducted an OTC transaction took 8 minutes to complete the transaction. Some of the users were able to map existing OTC interactions to what was happening on the phone, while others remained confused on domain knowledge, such as the difference between a PIN and Secret Code (explained in Phase Two Observations Section) and the uses of each. When asked what a PIN was, some participants mentioned that they did not know. Participants who mentioned knowledge about PIN gave examples of ATM PINS, phone lock code PINS, and EasyPaisa PINS. Thus, previous exposure to smartphones, their features, and DFS plays an important role in forming understanding and learnability of newer services including mobile wallets.

Mobile Money's Relevance and Convenience. Some participants mentioned the long travel distance to the closest bank branch or mobile money agent as a nuisance that could be overcome through a mobile wallet. A rural participant conjectured that, "I can transact from home and will not need to go to city after burning fuel worth 150 rupees and spending time as well. I can do something else in the same time. There is no mobile money agent shop in our nearby village but there is $4 G$ internet".

Participants divulged issues they had experienced while interacting with mobile money agents, such as lack of liquidity and agent fraud. Participants recounted requiring multiple visits over multiple days to one agent or visits to multiple agents to cash out complete amount of a transaction. One participant described a common scene: "If you go, there is a long line on the nearby small shops and they say they are out of cash. So I go to the bigger retailer shop in nearby village because they have a big business and they are not out of cash." Participants also shared that they have trust issues when paying bills using OTC transactions because the bill will show as unpaid on the following month's billing statement. Other times they will not receive a confirmation receipt.

Women and Mobile Wallets. Out of the 118 participants, 37 were women. Among these, 34 used phones (20 personal phones and 14 shared phones) and three had no phones. 29 of these women had received some education whereas five were illiterate. 15 of the total 37 women had used OTC transactions (12 for both sending and receiving money and three for receiving only). Ten women, all from urban areas, had bank accounts. None of the women had used mobile wallets.

Most women who did not send money cited that male members of their households handled all of the financial transactions. Sometimes young girls hesitated in admitting their interest in or previous use of smartphones or the Internet due to their families' perception of these technologies as not being good for girls. One woman explained that, "Its not considered good in our family for girls to use phones. Both my brothers (younger and elder) have smartphones. We do not have WiFi or cable since we cant afford it. They use someone else's WiFi who lives in our street."

\section{DISCUSSION}

Awareness about a product, following GSMA's (Groupe Speciale Mobile Association) customer journey model for mobile money [9], is considered the first step of the customer life cycle and a prerequisite for the customer to be able to understand, consider, compare, and adopt a product. We have observed varying levels of information challenges during our study, all of which hinder the adoption and use of mobile wallet applications. At the most basic level, we observed a lack of information and understanding over what a mobile wallet is, even for people who had done OTC transactions previously. While advertisements create awareness of mobile money brands, [12], the understanding of mobile money as a concept is often limited to OTC transactions. Mobile wallet 
accounts remain completely novel to many people. We observed a perceived lack of usefulness toward mobile money in general (OTC or wallet) beyond remittances. We propose the dissemination of information via focused messages to fill these gaps of knowledge, informing potential users of other mobile money payment options as well as opportunities for accessing savings, insurance, and credit through mobile wallets.

Trusted customer support centers are a great asset for telecoms to address user generated queries. Almost $50 \%$ of the participants mentioned calling the telecommunication company call center helpline for various reasons and demonstrated trust in the information provided by call agents. At the same time, participants expressed concern for their inability to discern an incoming customer support center call from a fraudulent one. We posit that this existing practice and credible knowledge source can be leveraged to efficiently inform users and should be integral in designing systems that address queries about mobile money applications. Some user concerns about call costs indicate that low-income customers can access these systems without hesitation when they are offered free of charge.

In addition to awareness and understanding, we identified information gaps regarding the trial of mobile apps. Barriers to mobile money app adoption include discomfort with new systems, lack of conceptual clarity regarding the origin of a financial account and the flow and source of funds, perceived risk of incorrect transactions and resulting financial loss, and lack of information on redress mechanisms for such wrong transactions. For low-income customers, the fear of financial loss becomes an adoption barrier as limited financial capability magnifies even small losses and outweighs any convenience benefits. OTC agents were preferred for the human element as well as offering an identifiable redress mechanism and clear responsibility in case of a wrong transaction. We propose that mobile money applications be made flexible enough to provide customers with options to retract incorrect transactions or, at the very least, provide a way to try the new system in a gamification environment without real ramifications. Such options, when clearly communicated and free of cost, can remove hesitation and encourage trial of mobile money applications, which results in increased learnability. Finally, we identified usage information gaps such as lack of understanding about key words like 'PIN code' and 'Secret code'. While secret codes are meant to be set by the sender, we learned that agents would set these codes themselves; this introduces the potential vulnerability of agents drawing from a limited combination of codes, leading to higher chances of fraud. To address this issue, we propose removing middle men and providing system generated random secret codes to share with senders and recipients through their mobile numbers.

\subsection{Women and the opportunity for increased independence}

In some of the households we visited, phone ownership was not defined by having either a feature phone or smartphone, but rather shared or multiple phone ownership arrangements. We witnessed households where men would leave their smartphones at home, opting for the feature phone to bring to work. This was observed amongst participants who were farmers, factory workers, or workers in environments where they feared that the smartphone may be damaged, lost, or stolen. This led to the potential for increased smartphone accessibility for women of the household for six to eight hours every day. We propose that such shared ownership models should be considered when designing solutions. Full time ownership of smartphone may not be necessary; women can be offered mobile wallet services over shared phones.

Most of the women we interviewed, after being informed about mobile wallet applications and gaining familiarity, concluded that mobile wallets would offer them an increased level of independence. They could make simple transactions - such as mobile top-ups and sending money - without leaving their homes or waiting for their husbands to return and complete transactions. However, they did not understand that cash-in transactions required traveling to a nearby shop to deposit cash, which could present issues with leaving the home.

\subsection{Design Considerations for Mobile Money}

We tested the use of audio help to decrease dependence on intermediated help for completing financial transactions. Previous research in ICTD has looked at intermediated technology use - the use of technology with the presence or help of an intermediary person to support the use [25] [8]. Audio help proved to decrease intermediated help required from the coach for low-literate users with no smartphone exposure. The use of side-by-side audio help proved to be more effective compared to tutorial videos as the ability of low-literate users to comprehend and store information about entire transaction is difficult. We observed that the audio help aids the user in traversing through the transaction flow interface, with little intermediated help, even with lack of exposure to application interfaces. However, for conceptual understanding of mobile wallet concepts, video help might be a better medium. We propose a combination of tutorial videos in the beginning for complex concepts and side-by-side audio help within the transaction flow.

We also posit that audio might not be the optimal or preferred way for help in financial services where secrecy or privacy of transactions is paramount. Our research shows that participants preferred the wallet because of its ability to maintain secrecy of the transaction, even from individuals in their immediate surroundings. Therefore, the users might not want to reveal the details of the transaction (sender, recipient, identity, and pin code) to everyone. In our research, interfaces with multiple options or menus per screen (the presence of various buttons or options hidden within deeper menus) presented complexities for participants, irrespective of age or literacy. In Phases Two and Three, some participants were unable to logically connect the progression from 'My Account' to 'Send Money'. In such cases, user demand-driven menus similar to those available in ATMs asking the user the action that they want to perform, might be better with a simple entrance into the main account.

\subsection{User Readiness to Adopt Mobile Wallets}

While we saw a lack of information about mobile wallet applications, we saw clear interest in using mobile money applications as this information gap was removed after initial introduction. It was encouraging to see the participants concluding the benefits of such applications based on their independent analysis just after 
the initial interaction with the application. Respondents said that it would be time-saving than OTC interactions. Female participants considered it a better option as they would not have to leave their homes to make financial transactions (they would still require a way to put cash in, but other transactions could be performed from home). The participants mentioned that the application was safe and secure because it would offer protection from pick-pockets and thieves. The participants also mentioned that the adoption of mobile wallets would enable more secrecy and security, since they could make transactions at home and in private (during OTC transactions everyone can see and hear the transaction).

During the study participants showed interest in using the mobile wallet application and asked questions about the application's name, release date, mobile money provider to release this application, transaction charges and availability on play store.

\section{CONCLUSION}

In this paper, we presented a three-phase learnability evaluation of smartphone-based mobile money applications in Pakistan. During the first phase, we tested an existing mobile money smartphone application and showed that low-literate users were unable to understand how to complete tasks in its complex UI. In the context of mobile money, learnability issues transcend usability concern and can act as adoption barrier. In the next two phases, working and testing with the prototype based on Karandaaz UI toolkit and later with the improved version, we saw that usability is an incremental process and found some further improvements. By adding side-byside audio help, we proved that audio help decreased the need for intermediated help by low-literate users with limited smartphone exposure in learning to use a mobile money app. We discuss that previous exposure or domain knowledge helps improves learnability and that metrics for learnability should be effectiveness and help sought, different from those of usability. A clear interest from people in using DFS application after testing showed that learnability is a deciding factor in whether a mobile money application can assist or stand in the way of financial inclusion.

\section{ACKNOWLEDGMENT}

We acknowledge the research grant from Financial Services for the Poor at the Bill and Melinda Gates Foundation that supported the work in this paper. We would like to thank Information Technology University (ITU) Administration especially Dr Umar Saif, Mr. Zaheer Sarwar and Mr. Syed Yasir Abbass, as well as Amna Batool, Tahir Ashraf and Kamran Bashir for their support. We also thank our collaborators and field partners, especially Akhuwat Microfinance Organization, its Board members and field officers for helping us in conducting the field research.

\section{REFERENCES}

[1] 2016. Smartphone User Interface and User Experience Design for Mobile Money in Pakistan. (Dec. 2016). http://www.karandaaz.com.pk/toolkit/demo-tool-kit/

[2] Akhuwat.org. 2012. Akhuwat - Journey of Hope. (2012). http://www.akhuwat. org.pk/index.asp

[3] Asli Demirguc-Kunt, Leora Klapper, Dorothe Singer, Peter Van Oudheusden. 2015. The Global Findex Database 2014 - Measuring Financial Inclusion around the World. (April 2015). http://documents.worldbank.org/curated/en/ 187761468179367706/pdf/WPS7255.pdf

[4] Banking Policy \& Regulations Department. 2007. Draft: Policy Paper on Regulatory Framework for Mobile Banking in Pakistan. (June 2007).
[5] Banking Policy and Regulations Department. 2016. Branchless Banking Regulations - For Financial Institutions desirous to undertake Branchless Banking. (July 2016). http://www.sbp.org.pk/bprd/2016/C9-Annx-A.pdf

[6] CGAP. The Power of Smartphone Interfaces for Mobile Money. (????). http: //www.cgap.org/blog/series/power-smartphone-interfaces-mobile-money

[7] CGAP. 2013. Mobile Money: OTC versus Wallets. (Sept. 2013). http://www.cgap. org/blog/mobile-money-otc-versus-wallets

[8] Sebastien Cuendet, Indrani Medhi, Kalika Bali, and Edward Cutrell. 2013. VideoKheti: Making Video Content Accessible to Low-literate and Novice Users. In Proceedings of the SIGCHI Conference on Human Factors in Computing Systems (CHI '13). ACM, New York, NY, USA, 2833-2842. DOI : http://dx.doi.org/10.1145/ 2470654.2481392

[9] Neil Davidson and M. Yasmina McCarty. 2011. Driving customer usage of mobile money for the unbanked. London, UK: GSM Association (GSMA) (2011). http://oauth2callback.gsma.com/mobilefordevelopment/wp-content/ uploads/2012/03/drivingcustomerusagefinallowres.pdf

[10] Asli Demirgüç-Kunt, Leora F. Klapper, Dorothe Singer, and Peter Van Oudheusden. 2015. The global findex database 2014: Measuring financial inclusion around the world. World Bank (April 2015). https://papers.ssrn.com/sol3/papers.cfm? abstract_id=2594973

[11] Alan Dix, Janet Finlay, Gregory Abowd, and Russell Beale. 1997. Human-computer Interaction. Prentice-Hall, Inc., Upper Saddle River, NJ, USA.

[12] Finclusion.org. Financial Inclusions Insights - FII - Driving Financial Inclusion Pakistan (Wave 2) Report. Technical Report. InterMedia. http://finclusion.org/ uploads/file/reports/InterMedia-FII-Pakistan-Wave-2-Wave-Report1.pdf

[13] Tovi Grossman, George Fitzmaurice, and Ramtin Attar. 2009. A Survey of Software Learnability: Metrics, Methodologies and Guidelines. In Proceedings of the SIGCHI Conference on Human Factors in Computing Systems (CHI '09). ACM, New York, NY, USA, 649-658. DOI : http://dx.doi.org/10.1145/1518701.1518803

[14] Karandaaz Pakistan. 2017. Karandaaz Pakistan - Smartphone UI \& UX Prototype Walkthrough. (2017). https://www.youtube.com/watch?v=4Pfp4quqZWI

[15] Mika Laakkonen. 2006. Learnability Makes Things Click: A grounded theory approach to the software product evaluation. Lapland University Press, Rovaniemi. https://core.ac.uk/download/pdf/30313005.pdf OCLC: 485260140.

[16] Dr Jonathan Lazar, Dr Jinjuan Heidi Feng, and Dr Harry Hochheiser. 2010. Research Methods in Human-Computer Interaction. John Wiley \& Sons. GoogleBooks-ID: H_r6prUFpc4C.

[17] Rock Leung, Joanna McGrenere, and Peter Graf. 2008. The learnability of mobile application interfaces needs improvement. In Proc. of British HCI Workshop on HCI and the Older Population. http://www.cs.ubc.ca/ rockl/downloads/ HCl08LeungMcGrenereGraf.pdf

[18] McKinsey Global Institute. 2016. Digital Finance For All: Powering Inclusive Growth In Emerging Economies. Technical Report. McKinsey\&Company. 122 pages. http://www.mckinsey.com/global-themes/employment-and-growth/ how-digital-finance-could-boost-growth-in-emerging-economies

[19] Indrani Medhi, Aman Sagar, and Kentaro Toyama. 2007. Text-free User Interfaces for Illiterate and Semiliterate Users. Inf. Technol. Int. Dev. 4, 1 (Oct. 2007), 37-50. DOI : http://dx.doi.org/10.1162/itid.2007.4.1.37

[20] Valerie Mendoza and David G. Novick. 2005. Usability over Time. In Proceedings of the 23rd Annual International Conference on Design of Communication: Documenting \& Designing for Pervasive Information (SIGDOC '05). ACM, New York, NY, USA, 151-158. DOI : http://dx.doi.org/10.1145/1085313.1085348

[21] Jakob Nielsen. 1993. Usability Engineering. Morgan Kaufmann Publishers Inc., San Francisco, CA, USA.

[22] Jakob Nielsen. 1996. Usability Metrics: Tracking Interface Improvements. IEEE Softw. 13, 6 (Nov. 1996), 12-13. http://dl.acm.org/citation.cfm?id=624616.625647

[23] Nigel Bevan. 1995. International Standards for HCI and Usability. Advances in Human Factors/Ergonomics 20 (July 1995), 885-890.

[24] Pakistan Telecom Authority. 2017. Telecom Indicators. (April 2017). http://www. pta.gov.pk/index.php?option=com_content\&task=view\&id=269\&Itemid $=658$

[25] Nithya Sambasivan, Ed Cutrell, Kentaro Toyama, and Bonnie Nardi. 2010. Intermediated Technology Use in Developing Communities. In Proceedings of the SIGCHI Conference on Human Factors in Computing Systems (CHI '10). ACM, New York, NY, USA, 2583-2592. DOI : http://dx.doi.org/10.1145/1753326.1753718

[26] Qasif Shahid, Lubna Razaq, Ahsan Mughal, Mahrukh Imtiaz, Myra Piracha, and Omar Shahid. SEEDING INNOVATION A framework for rooting FinTechs in Pakistan. Technical Report. FinSurgents. http://www.karandaaz.com.pk/wp-content/ uploads/2017/01/Seeding-Innovation.pdf

[27] State Bank of Pakistan. 2011. State Bank revises Branchless Banking Regulations to bring low income segment of society into financial loop. (June 2011). http: //www.sbp.org.pk/press/2011/Regulations-20-Jun-11.pdf

[28] Ravi Vatrapu, Dan Suthers, and Richard Medina. 2008. Usability, sociability, and learnability: A CSCL design evaluation framework. In Proceedings of the 16th international conference on computers in education (ICCE 2008). http://www. academia.edu/download/30702845/ICCE2008-paper242.pdf

[29] Susan Weinschenk. 2011. 100 Things Every Designer Needs to Know About People (1st ed.). Pearson Education. 